elicited perceptions of: long-term symptoms attributed to injury, influences on recovery, outstanding needs for support, and opportunities to extend current health and social resources. Interviews were audio-recorded, transcribed, organized in NVIVO, and analyzed using inductive-deductive thematic analysis.

Results Four overarching themes emerged. The first, 'you used to run' includes subthemes signifying the persistence of psychological distress, chronic pain, increased substance use, changes in sleep, and social isolation 12-36 months after injury. The second, 'where it happened,' describes the impact of neighborhood recovery environments on traumatic stress, gun carrying, outdoor activity, and proximity to social networks. The third, 'in a hole,' refers to post-injury changes in workforce participation, financial stability, and barriers to healthcare access. The fourth, 'should be somebody's job to help' included perceived needs for services that address workforce re-entry, psychological stress, healthcare access and peer group interaction.

Conclusions The perceptions of Black men in Philadelphia on their long-term recovery illustrates individual, environmental, and structural factors that can be targeted for tertiary prevention.

Contributions to Injury and Violence Prevention Science Qualitative evaluation of the effects of trauma can be used to identify pathways through which to diminish outcome disparities and their origins in the socio-ecological context of recovery.

\section{Epidemiology of TBI}

\section{TRAJECTORY OF SALIVARY MIRNA GENE EXPRESSION IN CHILDREN WITH CONCUSSION}

Lindsay Sullivan, Amanda Hautman, Jingzhen Yang. ${ }^{~}$ Center for Injury Research and Policy at The Abigail Wexner Research Institute at Nationwide Children's Hospital

\subsection{6/injuryprev-2020-savir.77}

Purpose Up to one-third of children with concussion develop persistent post-concussion symptoms (PPCS), which can give rise to subsequent problems for years post-injury. This pilot study aimed to (1) monitor gene expression of a panel of salivary miRNAs over time post-concussion and (2) identify biomarkers that differentiate concussed children who develop PPCS from those who do not.

Methods Saliva samples were collected from concussed children ages 11-17 at $\leq 1$ week, 2-weeks, and 4-weeks postinjury. Post-concussion symptoms were also monitored daily throughout enrollment. PPCS was defined as a symptom score of 5 or higher than pre-injury level beyond 28 days postinjury. Changes in salivary miRNA expression over time postinjury were analyzed using the NanoString nCounter ${ }^{\circledR}$ human v3 miRNA panel to compare children with and without PPCS.

Results Of the 23 children studied (64 saliva samples provided), the average age was 14.4 years, $52.2 \%$ were male, and $34.7 \%(n=8)$ experienced PPCS. Of the 798 different human miRNAs analyzed, 46 (5.8\%) had gene expression levels above threshold in at least $75 \%$ of the saliva samples analyzed. Six miRNAs that were differentially expressed in the PPCS group were significantly under expressed within one week of injury $(\mathrm{p}<0.05)$. Compared to children without PPCS, children with PPCS showed significant changes in 18 miRNA expression levels from within one week to 2-weeks post-injury (17 increased, $p<0.05 ; 1$ decreased, $p<0.01$ ). Significant changes were also observed in 21 miRNA expression levels from 2 weeks to 4-weeks post-injury (19 miRNAs decreased, $\mathrm{p}<0.05$; 2 miRNAs increased, $\mathrm{p}<0.05)$.

Conclusion Additional studies are needed to verify our findings and to examine the biological regulatory or inflammatory mechanisms of the differently expressed miRNAs.

Significance The identification of clinically-relevant biomarker miRNAs could inform the development of individually-tailored treatment plans for children at risk for PPCS.

\section{Violence research and prevention in healthcare settings}

\section{SHIFTING DEMOGRAPHICS: 14-YEAR TRENDS IN VIOLENT INJURIES AT A LEVEL I TRAUMA CENTER}

${ }^{1}$ Maxwell Presser, 'Adaobi Nwabuo, '2Lilian Li. 'University of California, San Francisco, University of Miami Miller School of Medicine; ${ }^{2}$ San Francisco Department of Public Health

\subsection{6/injuryprev-2020-savir.78}

Statement of Purpose This study analyzes changes in sociodemographic and clinical characteristics of violently injured patients to guide injury prevention strategies.

Methods/Approach Trauma registry data from 2005 to 2018 was analyzed to identify trends among victims of community violence. This hospital is the only level I trauma center in a large, urban area, treating 99\% of all violently injured patients in the city.

Results Since 2005, the median age of violently injured patients increased 9 years from 26 to 35 . During this same timeframe, there was an $8.2 \%$ increase in violent injury among the White population, a $6.3 \%$ increase among the Latinx population, and a $4.9 \%$ decrease among the Black population. The rate of severe injury per 100,000 decreased from 118 to 86 in the White population and 325 to 212 in the Black population but increased from 267 to 305 in the Latinx population. There was an $18.5 \%$ decrease in penetrating injuries and $18.5 \%$ increase in blunt injuries. The mean length of hospital stay following violent injuries increased by 4.1 days.

Conclusions The age of violently injured patients is increasing, and the demographic makeup of violently injured patients is shifting to include a greater proportion of White and Latinx individuals. During the study period, the proportion of Black residents in the city decreased by $28 \%$, but the observed decrease in violent injury in this population was much lower. Additionally, the percentage of Latinx residents in the city increased by $8 \%$ during this time, but violent injury increased disproportionately.

Significance and Contributions to Injury and Violence Prevention Science As older populations may have different risk factors, understanding these trends is essential to ensure violence prevention programs provide appropriate interventions and can adapt to effectively target the older population. Prolonged hospital stays may extend the opportunities for interventions to be offered. 